\title{
Advancement of the 10-species subgingival Zurich Biofilm model by examining different nutritional conditions and defining the structure of the in vitro biofilms
}

\author{
Thomas W Ammann*, Rudolf Gmür and Thomas Thurnheer
}

\begin{abstract}
Background: Periodontitis is caused by a highly complex consortium of bacteria that establishes as biofilms in subgingival pockets. It is a disease that occurs worldwide and its consequences are a major health concern. Investigations in situ are not possible and the bacterial community varies greatly between patients and even within different loci. Due to the high complexity of the consortium and the availability of samples, a clear definition of the pathogenic bacteria and their mechanisms of pathogenicity are still not available. In the current study we addressed the need of a defined model system by advancing our previously described subgingival biofilm model towards a bacterial composition that reflects the one observed in diseased sites of patients and analysed the structure of these biofilms.
\end{abstract}

Results: We further developed the growth media by systematic variation of key components resulting in improved stability and the firm establishment of spirochetes in the 10-species subgingival Zurich biofilm model. A high concentration of heat-inactivated human serum allowed the best proliferation of the used species. Therefore we further investigated these biofilms by analysing their structure by confocal laser scanning microscopy following fluorescence in situ hybridisation. The species showed mutual interactions as expected from other studies. The abundances of all organisms present in this model were determined by microscopic counting following species-specific identification by both fluorescence in situ hybridisation and immunofluorescence. The newly integrated treponemes were the most abundant organisms.

Conclusions: The use of 50\% of heat-inactivated human serum used in the improved growth medium resulted in significantly thicker and more stable biofilms, and the quantitative representation of the used species represents the in vivo community of periodontitis patients much closer than in biofilms grown in the two media with less or no human serum. The appearance of T. denticola, P. gingivalis, and T. forsythia in the top layer of the biofilms, and the high abundance of $T$. denticola, reflects well the microbial situation observed at diseased sites. The improved model biofilms will allow further investigations of interactions between individual species and of the effects of atmospheric or nutritional changes, as well as interactions with tissue cells.

Keywords: Periodontitis, Model, Biofilm, Subgingival, Structure, CLSM, FISH, IF

\footnotetext{
* Correspondence: thomas.ammann@zzm.uzh.ch

Section of Oral Microbiology and Immunology, Institute of Oral Biology,

Center of Dental Medicine, Plattenstrasse 11, 8032 Zürich, Switzerland
} 


\section{Background}

In the oral cavity, bacteria encounter many different stress factors. Shear-forces and high flow rates of saliva dominate on exposed surfaces, while bacteria colonizing the gingival crevices and/or subgingival pockets have to contend and withstand with the host's immune response. As in most other environments, bacteria form biofilms as protection from these harsh conditions [1].

The bacterial community colonizing the oral cavity is highly complex and varies considerably between different individuals. According to current reports, 600 to 700 established species and likely several thousand only partially cultivable taxa can be detected [2]. However, this consortium does not pose a threat to a healthy individual. It even has a protective function by preventing the establishment or predominance of harmful organisms [3]. Several factors like imbalanced nutrition, smoking, diabetes, emotional stress, or genetic predisposition [4] can lead to changes in the composition of this subgingival community, leading to a loss of the natural ecological balance. Potentially pathogenic species may increase in numbers, starting to cause persistent infections of host tissues that are capable to cause not only tooth loss and bone resorption but also can spread out to extra-oral sites and become systemic [5]. Physical removal of the biofilms, possibly followed by an antibiotic treatment in refractory cases, is used to re-establish a biofilm community compatible with clinical stability. However, treatment will never and should not remove all organisms, since this could lead to settlement of even more harmful organisms. It is an almost impossible task to identify and selectively target only the actual pathogens among the hundreds of different species present [6].

Out of the potentially thousands of species found in the oral cavity, about 400 can be detected in periodontal pockets. This number is reduced to a range of 100 to 200 species in one patient [7]. The enormous diversity makes subgingival biofilms difficult to study and it seems impossible to fully understand all the interactions between the species. To investigate and better understand the role of individual species, models reflecting subgingival colonization are needed. Regarding the sophisticated structure of these biofilms [8], it is obvious that biofilms consisting of only one or two organisms do not sufficiently mirror the in vivo situation. Some investigators solved this problem by using inocula taken from diseased sites of patients $[9,10]$. Major problems in such model systems are both the restricted possibilities for analysis of all species involved and the composition of the inoculum, which inevitably varies substantially between donor patients.

An in vitro model system for subgingival biofilms should not only be functional in terms of pathogenic potential, it should also have a defined structure and a quantitative relationship between the species that resemble to some extent the in vivo situation. The aim of this study was therefore to further develop our 10-species model system [11] by 1) incorporating treponemes and balancing the growth medium to optimize their growth and 2) defining the structure of the produced biofilms. The incorporation of Treponema denticola, replacing Treponema lecithinolyticum used in our previous study, along with the variation of the growth medium allowed the treponemes to firmly establish in the biofilms. Further, F. nucleatum subsp. vincentii KP-F2 (OMZ 596), Campylobacter rectus (OMZ 697), Streptococcus intermedius ATCC 27335 (OMZ 512) were replaced by better growing strains (see methods).

The described modified model provides the possibility to examine the impact of variable growth conditions as well as the role of individual species. The high complexity of our 10-species model provides biofilms that are much closer to the in vivo situation than other models using just one or two species.

\section{Results}

\section{Development of biofilms}

Three different growth media were compared regarding bacterial abundances and biofilm stability: SAL (60\% pooled, heat inactivated saliva, 30\% modified fluid universal medium containing $0.3 \%$ Glucose [mFUM; [12]] and $10 \%$ heat-inactivated human serum), mFUM4 (100\% mFUM containing $4 \mathrm{mM}$ glucose), and iHS (50\% heat-inactivated human serum and 50\% mFUM with 4 $\mathrm{mM}$ glucose. They all had low final concentrations of glucose: $4 \mathrm{mM}$ for iHS and mFUM4, and $5.5 \mathrm{mM}$ for SAL respectively. The formation of the biofilms was observed by determination of total counts on Columbia blood agar (CBA) plates at 5 time points during the incubation time. The final structure, as well as the thickness of the biofilms at 5 time points during the incubation time, was determined by confocal laser scanning microscopy (CLSM). The experiments confirmed and extended our previous finding [11] that the composition of the growth medium has a major effect on the development, stability and composition of the biofilms.

The iHS medium delayed biofilm formation by $20 \mathrm{~h}$ compared to mFUM4 (Figure 1). $4 \mathrm{~h}$ after inoculation in mFUM4, the discs were densely colonized by cocci. Based on the observation that most of these cocci appeared as chains, they can be assumed to be streptococci. However, after $4 \mathrm{~h}$ of incubation in iHS, cocci were observed to appear almost exclusively as dense microcolonies, while rods (morphologically Fusobacterium nucleatum, Prevotella intermedia, or Tannerella forsythia) in low abundance colonized the majority of the disc. Incubation in SAL medium led to a similar 


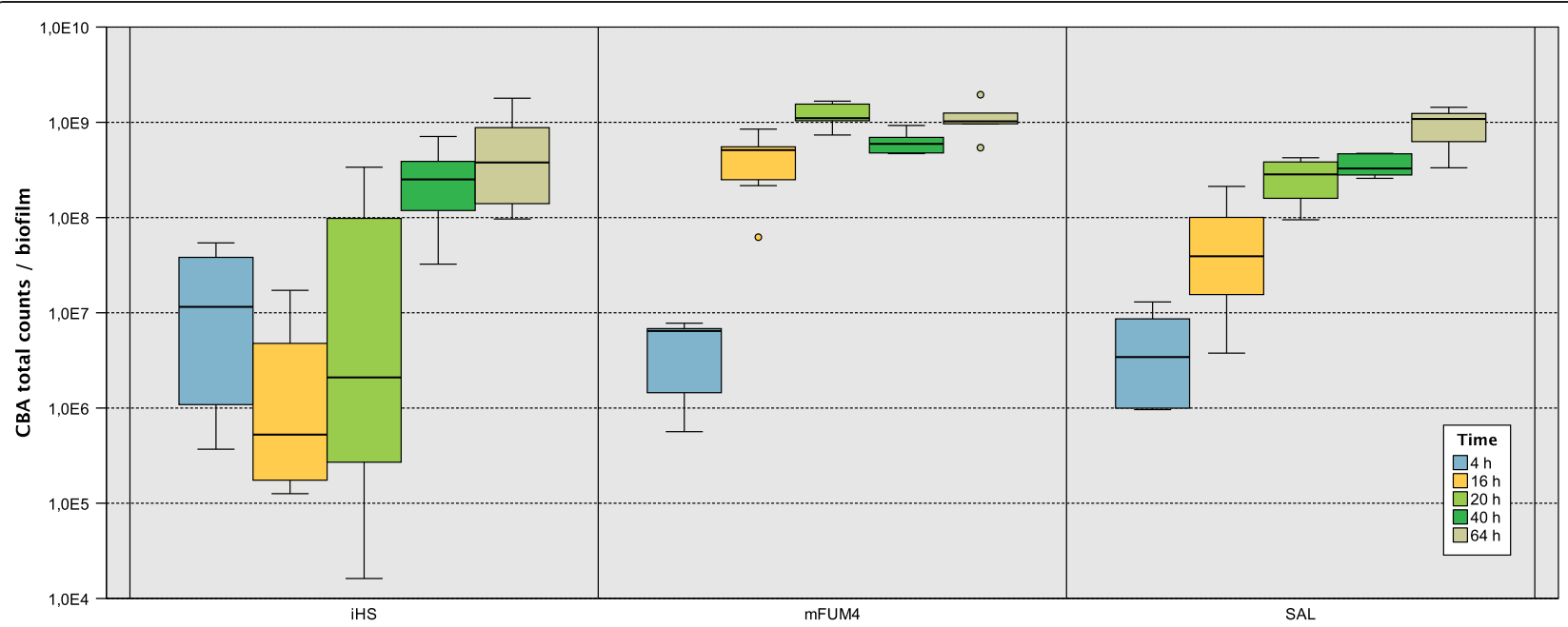

Figure 1 Time course of biofilm growth comparing SAL, mFUM4, and iHS as growth media. Total counts determined by plating on CBA agar plates ( $T$. denticola and $T$. forsythia are not cultivable on CBA). Each box represents $N=9$ independent biofilms from three independent triplicate experiments. The boxes represent the inter quartile range of the data points, the bar indicates the median. The whiskers cover the data points within the $1.5 x$ inter quartile range. Dots are outliers within 1.5 and 3 box lengths outside the interquartile range.

observation as in mFUM4: The disc was colonized mainly by cocci (Figure 2).

The high concentration of human serum in iHS improved biofilm stability in terms of firm attachment to the disc (less cell loss during dip washing and the FISH staining procedure), and further the average thickness of the biofilms was significantly increased after $64.5 \mathrm{~h}$ when compared to biofilms grown in mFUM4, or SAL respectively (Figure $3 \mathrm{~A}$ ). However, the total counts of bacteria per biofilm did not show significant differences between the three growth media (Figure 3B).

In mFUM4, biofilms showed a rapid increase in biofilm thickness and total counts right after inoculation and reached their highest cell numbers after $20 \mathrm{~h}$. While stable until then, they tended to partially detach from the discs during the dip-washes at later time points. In contrast, major parts of biofilms grown in iHS detached during the dip-washes in the first $20 \mathrm{~h}$ of incubation. This observation is in accordance with the strong decrease in total counts along with a high variability between different experiments and replicates. During further incubation, however, the remaining parts had stabilized and the biofilms showed a rapid increase in thickness and total counts. Biofilms cultivated in SAL medium showed a constant increase of total counts and thickness and were not prone to detachment during the incubation time (Figure 1).

\section{Quantitative representation of species in biofilms}

We determined the cell numbers of all organisms in biofilms grown either in SAL, mFUM4, and iHS medium. Enumeration of cells was performed by microscopical counting following staining the bacteria by fluorescence in situ hybridisation (FISH) or immunofluorescence (IF). The data are summarized in Figure 4. Treponema denticola showed significantly higher cell numbers in iHS compared to SAL and mFUM4 and was among the most abundant organisms in the biofilm. In mFUM4, Treponema denticola hardly proliferated and only appeared in abundances close to the detection limit. Streptococcus anginosus and Veillonella dispar showed significantly reduced growth in SAL medium compared to the other two media, while Actinomyces oris showed significantly reduced growth in iHS compared to mFUM4.

The abundances of Streptococcus oralis, F. nucleatum, Campylobacter rectus, P. intermedia, Porphyromonas gingivalis, and $T$. forsythia were not affected by the growth medium.

\section{Structure of mature biofilms}

The quantitative representation of the used species was most convincing when biofilms were grown in iHS medium. T. denticola established in high numbers and the biofilms showed the best stability during the following staining procedures. Therefore, structural analysis was focused on these biofilms. CLSM analyses of FISH stained biofilms enabled us to determine all 10 species used in the model and locate their position in the biofilms. The top layer (approximately $30 \mu \mathrm{m}$ from the biofilm surface) and basal layer (approximately $50 \mu \mathrm{m}$ from the disc surface) of the biofilms showed clear structural differences and a fluent transition between these layers was observed. Biofilms grown in mFUM4 showed a dominance of $F$. nucleatum and streptococci in the basal layer (Figure 5A). In biofilms grown in iHS, however, $F$. nucleatum was detectable by FISH only in the top layer 
Figure 2 Bacterial attachment to the disc surface under different nutritional conditions $\mathbf{4} \mathrm{h}$ after inoculation.

Comparison of the growth media mFUM4 (A), iHS (B) and SAL (C). green: DNA staining using YoPro-1 + Sytox. The disc surface is visualized in grey colour. The images show representative areas of one disc each. Scale bars: $15 \mu \mathrm{m}(\mathrm{A} / \mathrm{B})$ and $10 \mu \mathrm{m}(\mathrm{C})$.

as dispersed cells, while streptococci were very abundant throughout the whole biofilm (Figure 5B). Aggregations of streptococci were often mixed with $V$. dispar in the whole biofilm except in the top layer, where $V$. dispar occurred as compact microcolonies (Figure 6). In biofilms grown in mFUM4, which had a lower thickness, this growth pattern of $V$. dispar was observed throughout the biofilm (Figure 5A). P. intermedia was found predominantly in the lower half of the biofilms forming microcolonies with diameters of about $50 \mu \mathrm{m}$ on average (Figure 7A). T. forsythia was found mainly in the top layer of the biofilm, while none were detected in the lower half of the biofilms (Figure 7A). T. denticola grew loosely in the top layer alongside with $P$. gingivalis, which displayed the highest density in close proximity to T. denticola accumulations (Figure 7B). A. oris appeared as loose EPS-embedded microcolonies located in the upper half of the biofilms (Figure 8A). Campylobacter rectus was dispersed throughout the biofilm and did not form own microcolonies, but showed higher density in the top layer of the biofilm (Figure 8B).

\section{Discussion}

This study focused on the importance of the nutritional conditions and the structure of subgingival biofilms generated on HA discs in vitro. The alteration of the growth medium by eliminating saliva and increasing the concentration of heat-inactivated human serum affected the biofilms positively as they developed to higher thickness, were more stable and enabled the extensive proliferation of $T$. denticola, which were observed only in small numbers using media with low or no heat-inactivated human serum. We were able to locate all the 10 organisms by multiplex FISH in combination with CLSM. The biofilms displayed a stratified structure reminiscent of in vivo subgingival biofilms [13]. However, in contrast to the in vivo situation, $F$. nucleatum was predominant in the basal layer along with streptococci of the biofilms grown in mFUM4. In biofilms cultured in iHS, F. nucleatum was detected as dispersed cells in the top layer. Earlier experiments showed that $F$. nucleatum has a strong dependency on streptococci, and is only able to establish along with them (data not shown). This observation is in accordance with the finding of co-aggregation studies that identified the ability of streptococci to attach to components of the pellicle, 


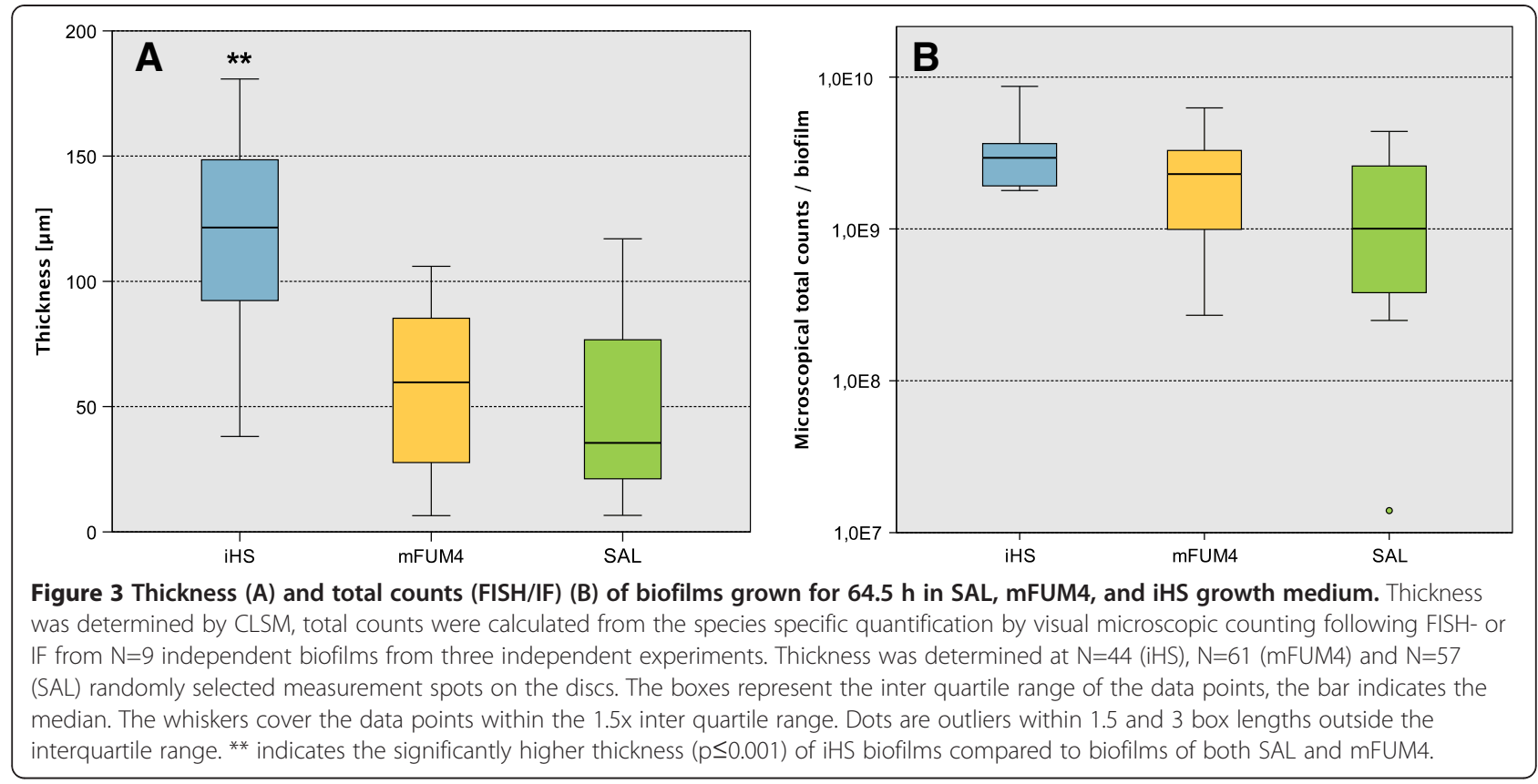

while $F$. nucleatum was shown to bind to the streptococci and act as a "bridging organism" for other species to colonize the biofilm [14]. The observed difference that $F$. nucleatum establishes in the basal layer might very well be due to the fact, that all strains were inoculated simultaneously. If no streptococci were added to the inoculum, but added to the biofilms at a later time point, $F$. nucleatum did not establish in the basal layer but rather after the addition of the streptococci, forming an intermediate layer. In this case, mainly $A$. oris was detected as an early colonizer (data not shown). Possibly, it would make sense to add the various strains sequentially, simulating the shift from health to disease.

The growth medium affected not only the biofilm composition; it had a strong influence on the rate of biofilm formation as well. The observed delay of biofilm formation in iHS medium could be explained possibly by the blocking of adhesion receptors on the saliva pellicle. It was shown for Streptococcus pneumonia and Escherichia coli that albumin inhibits biofilm formation on various surfaces $[15,16]$. It is very likely that this effect also occurs in our model during colonization of the

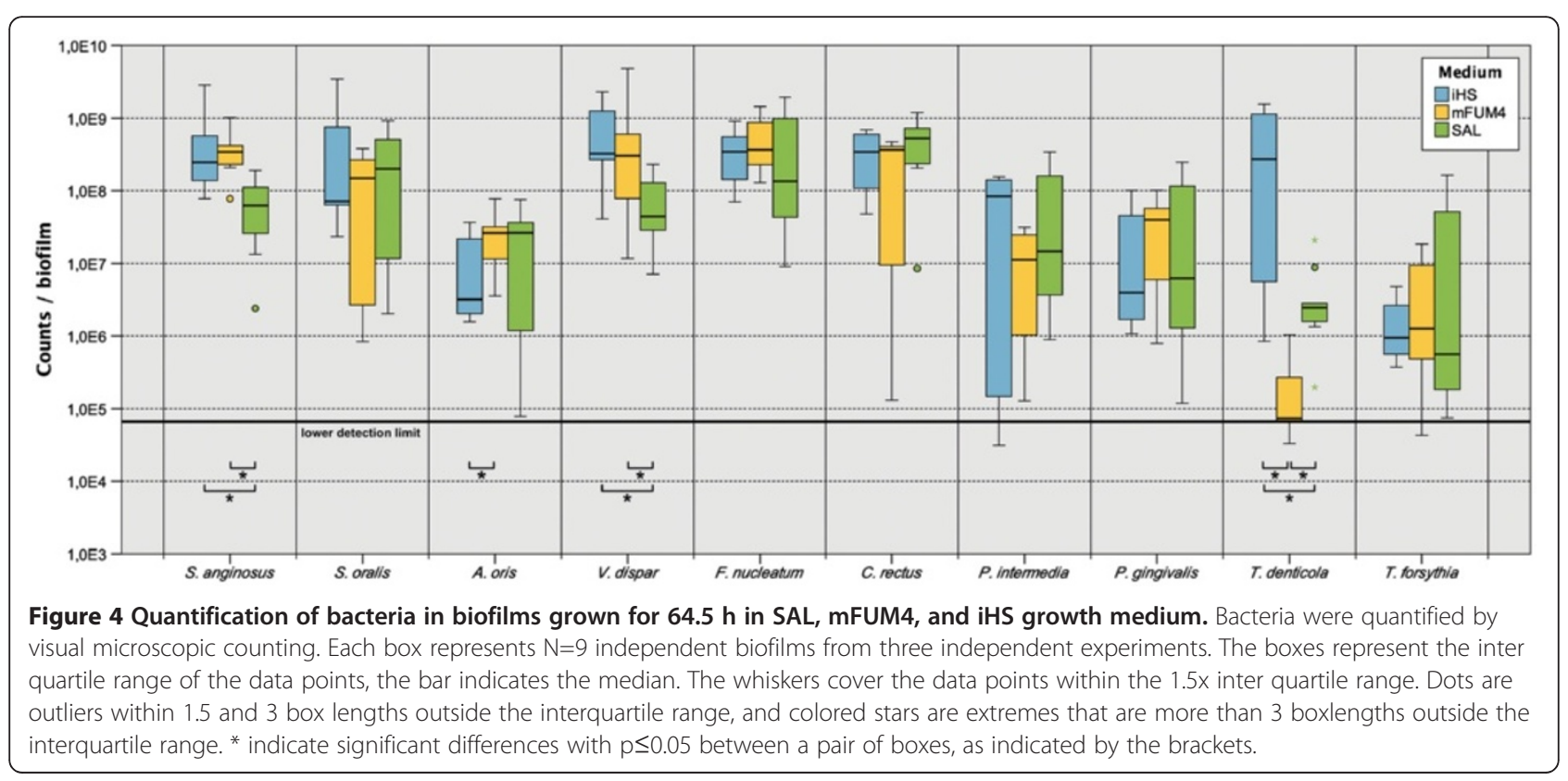




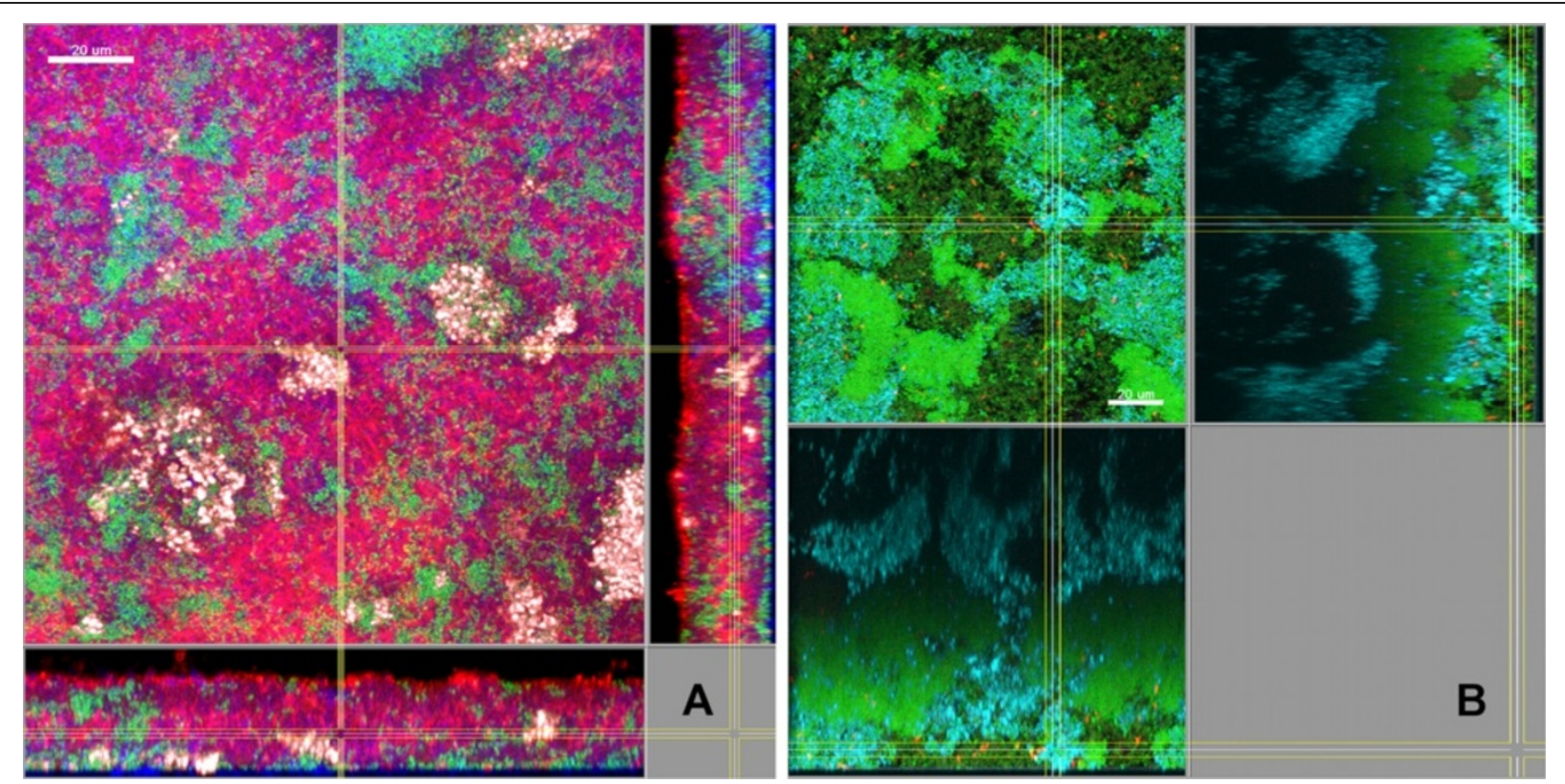

Figure $\mathbf{5}$ Biofilms grown for $\mathbf{6 4 . 5} \mathbf{h}$ in or mFUM4- (A) or iHS medium(B). FISH staining of a fixed biofilm; the biofilm base in the side views is directed towards the top view. (A) red: F. nucleatum, white: V. dispar, green: non-hybridised cells, DNA staining (YoPro-1 + Sytox), blue: EPS. (B) cyan: streptococci, red: F. nucleatum, green: non-hybridised cells, DNA staining (YoPro-1 + Sytox). Figures show a representative area of one disc. Scale bars: $20 \mu \mathrm{m}$.

discs. However, even though the initial attachment of the bacteria is prevented to a certain degree, all ten organisms were able to persist on the discs and were not washed away during dip-washing. Independent of the used medium, the biofilms showed a phase with a pronounced increase in thickness and bacterial abundance. This phase took about $20 \mathrm{~h}$ regardless of the used medium, however, the medium does affect its onset. Concluding this, it seems that a certain number of bacteria attached to the disc is required to promote "exponential" biofilm formation. Our experimental setup did not allow defining the reason(s) behind this phenomenon. Possibly, it is triggered by quorum sensing, as it was shown for several oral species that AI-2 or CSP signalling is involved in biofilm formation [17]. Alternatively, it could be that early biofilm formation under different nutritional conditions leads to different degrees of biofilm rigidity and therefore to different levels of sensitivity to shear-forces applied during biofilm dip-washing.

The iHS medium produced significantly higher cell numbers of $T$. denticola per biofilm compared to mFUM4 or SAL medium. However, $P$. gingivalis and $T$. forsythia were not affected by the higher serum concentration. This is surprising, since $P$. gingivalis was reported to profit from gingival crevicular fluid as well as from menaquinone secreted by veillonellae [17], and since one of the main growth factors of T. forsythia, Nacetyl-muramic acid [18], should be plenty available in thicker biofilms with probably increased proportions of lysing cells. On the other hand both species are known to be quite fastidious and our data indicate that it will be necessary to optimize further media components to increase their growth rates. S. anginosus, A. oris, and $V$. dispar showed mathematically significant reactions to the different growth media as well. However, in neither case the differences were greater than one log, which can hardly be considered as "biologically significant".

The biofilms proliferating in iHS medium showed a consistent structure throughout the replicates and the organisms showed interactions as they could be expected according to literature. Zjinge et al. described three different layers in in vivo subgingival samples [13]. Our model biofilms showed differences between topand basal layers as well, however, it was not possible to clearly define an intermediate layer. It rather seems that there is a fluent transition between top- and basal layer of the biofilms. The two layers show distinct characteristics. In the basal layer, biofilms were very compact and contained mainly streptococci, some veillonellae and large amounts of $F$. nucleatum and $P$. intermedia. The presence of $P$. intermedia was unexpected as it is in contrast to the in vivo situation where coccoid Prevotella species preferentially colonize the top layer in form of compact microcolonies [13]. The top layer of the model biofilms showed a rather loose structure with a lot of EPS. $V$. dispar and other cocci were embedded as compact microcolonies in their matrix, while $A$. oris 


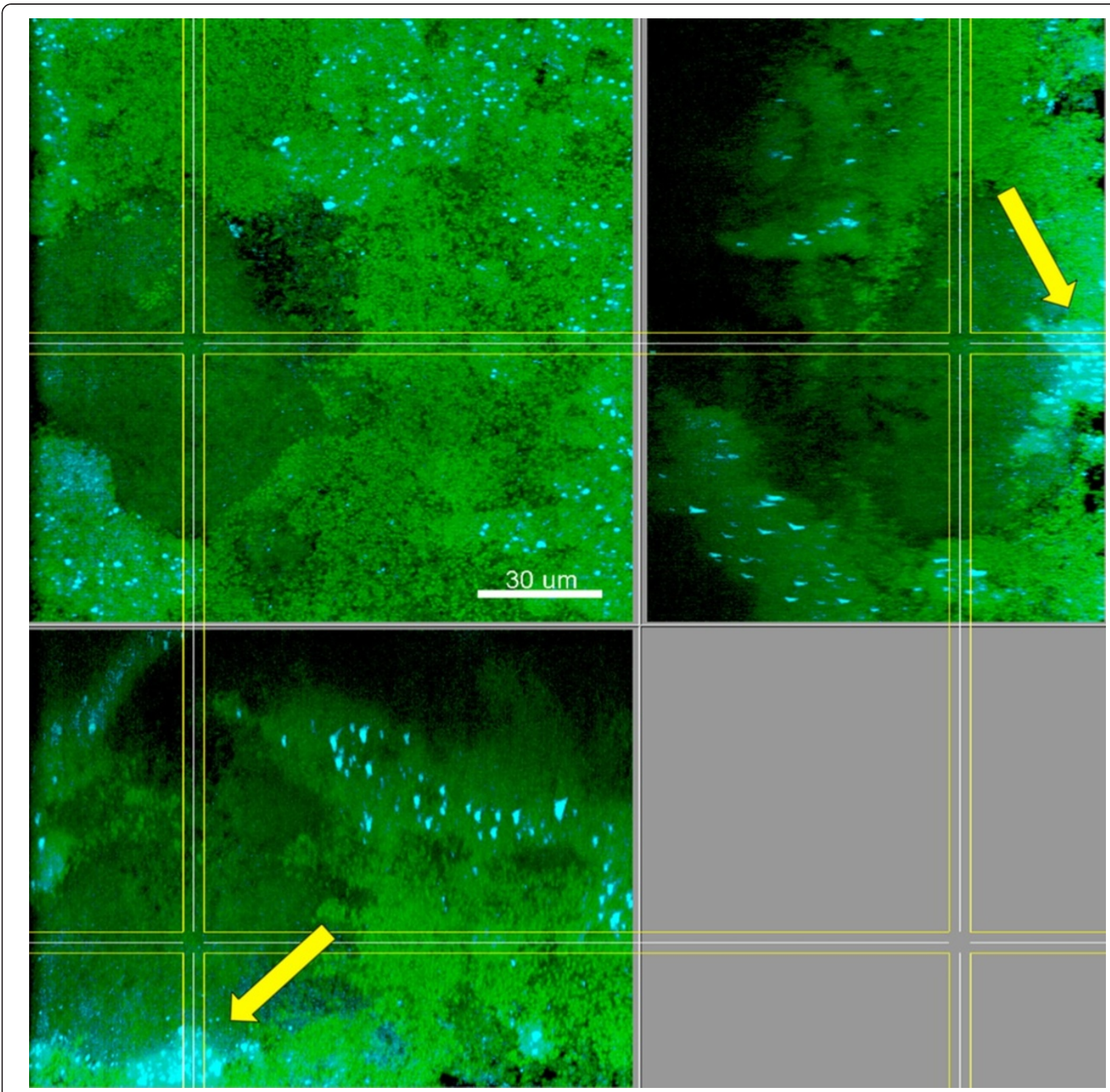

Figure 6 Biofilms grown for $\mathbf{6 4 . 5} \mathbf{~} \mathbf{~ i n ~ i H S ~ m e d i u m . ~ F I S H ~ s t a i n i n g ~ o f ~ a ~ f i x e d ~ b i o f i l m ; ~ t h e ~ b i o f i l m ~ b a s e ~ i n ~ t h e ~ s i d e ~ v i e w s ~ i s ~ d i r e c t e d ~ t o w a r d s ~ t h e ~}$ top view. Cyan: $V$. dispar, green: non-hybridised cells, DNA staining (YoPro-1 + Sytox). Arrows: Microcolonies of $V$. dispar. Shown is a representative area of one disc. Scale bar: $30 \mu \mathrm{m}$.

appeared as loose microcolonies, with EPS surrounding each cell. In some preliminary diffusion experiments, similar to these described by Thunheer et al. for in vitro built supragingival biofilms [19], it seemed that these loose regions might work as diffusion channels, allowing large molecules to reach the basal layer in less than two minutes (data not shown).

The high abundance of $T$. denticola along with $P$. gingivalis and $T$. forsythia in the top layer was remarkable. The location, combined with the known high pathogenic potential of these species, might indicate a high inflammatory potential of our model biofilms. Particularly striking was to find $T$. denticola and $P$. gingivalis to colonize in close proximity, indicating some sort of metabolic dependency. This observation corresponds well with several previous studies. For example, it has been shown in a murine abscess model that the pathogenicity of $P$. gingivalis was significantly increased in presence of $T$. denticola [20]. The result was recently confirmed in a murine alveolar bone loss model, where co-inoculation showed a strong response not only for bone loss, but also for $P$. gingivalis specific $\mathrm{T}$ cell proliferation and interferon $-\gamma$ 


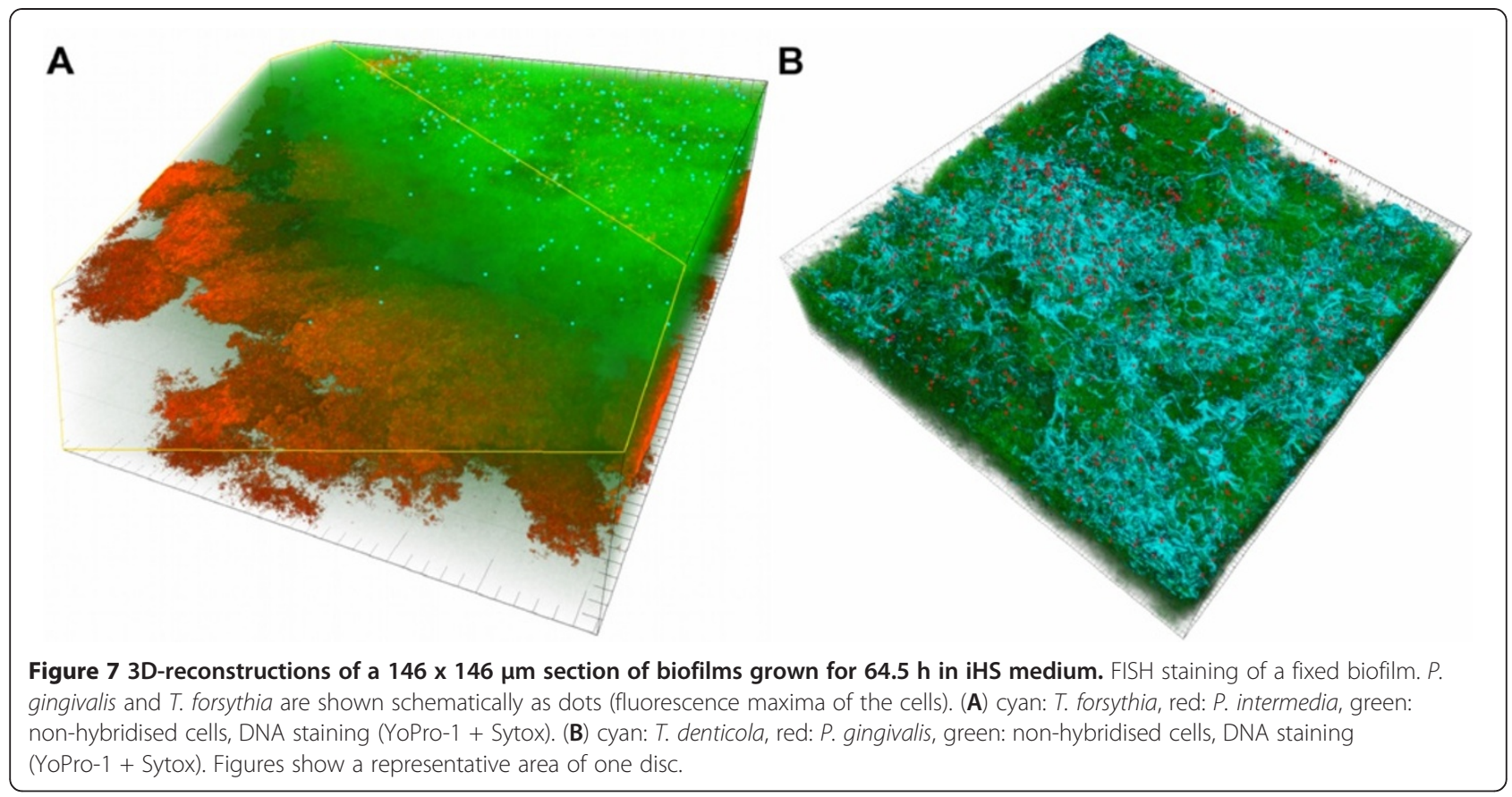

production [21]. And in yet two other studies $P$. gingivalis and $T$. denticola had shown metabolic synergies by exchanging iso-butyric- and succinic acid [22] and an ability to co-aggregate with the Hgp44 domains of RgpA, Kgp and HagA acting as the key adhesins [23]. Other organisms found in this study in highest density in the top layer but without a specific focal distribution were C. rectus, $F$. nucleatum and T. forsythia. In the case of $C$. rectus, a highly motile microaerophilic organism, this meets the expectation. In biofilms grown in iHS medium, it was not possible to detect dense colonies of $F$. nucleatum in the basal layer by FISH, as it was the case in thin mFUM4

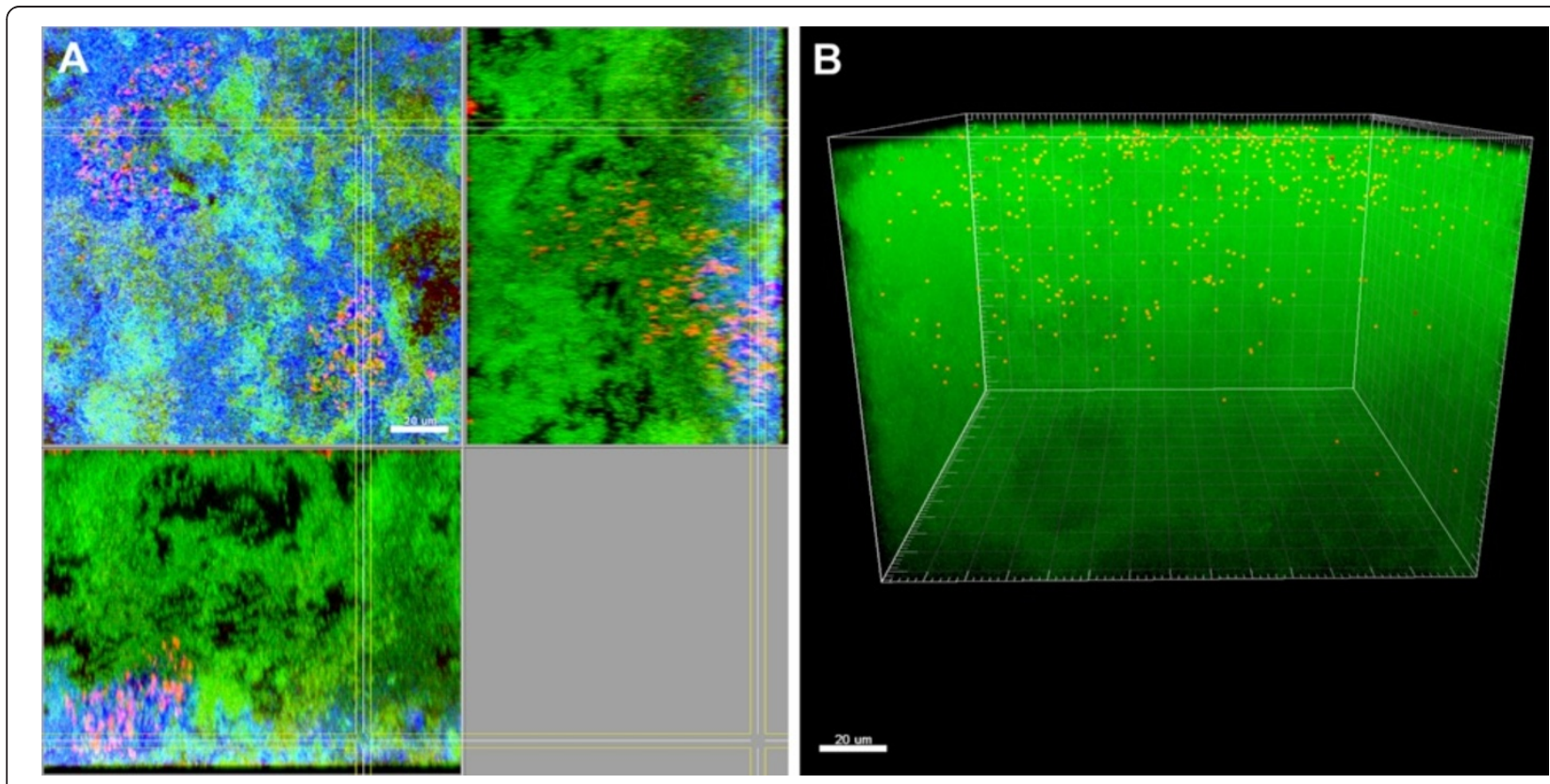

Figure 8 Biofilms grown for $\mathbf{6 4 . 5} \mathbf{~}$ in iHS Medium. FISH staining of a fixed biofilm; the biofilm base in the side views is directed towards the top view. C. rectus is shown schematically as dots (fluorescence maxima of the cells). (A) red: A. oris, green: non-hybridised cells, DNA staining (YoPro-1 + Sytox), blue: EPS. (B) red: C. rectus, green: non-hybridised cells, DNA staining (YoPro-1 + Sytox). The red dots appear yellowish due to the transparency of the green channel. Figures show a representative area of one disc. Scale bars: $20 \mu \mathrm{m}$. 


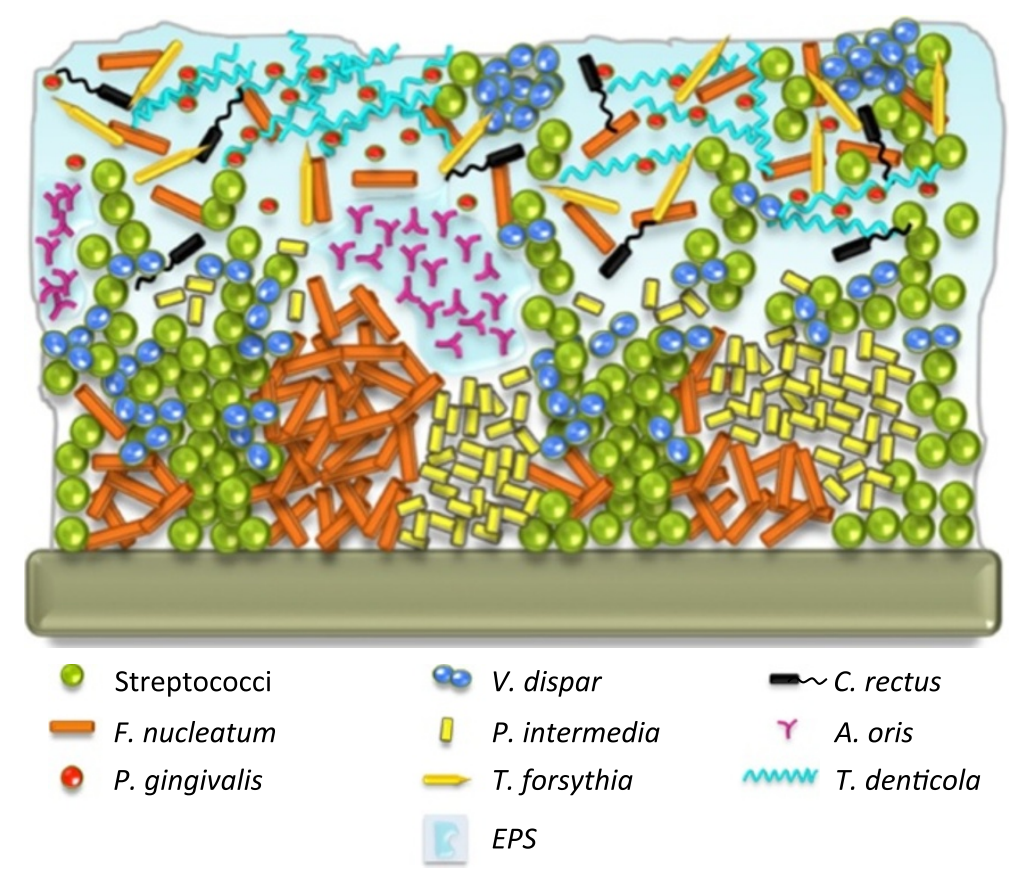

Figure 9 Schematic structure of the 10-species in vitro biofilms after $64 \mathrm{~h}$ of incubation in iHS medium. Distribution of the 10 species and EPS as observed by CLSM. The scale is not representative.

biofilms. There are several factors that could explain this finding. On the one hand, Sharma et al. made the same observation in two species biofilms of $F$. nucleatum and $T$. forsythia. Using a live-dead staining, they found mainly non-viable $F$. nucleatum attached to the substratum, while the bacteria in the upper layer of the biofilms showed a high viability [24]. Further, they observed synergistic growth of these organisms, which could explain the occurrence of $T$. forsythia together with the active $F$. nucleatum in the top layer of our biofilms. On the other hand, the effect could be explained by known problems of FISH staining procedure: The probes or the fixative possibly were not able to diffuse through the whole biofilm, or cells had strongly reduced number of ribosomes, leading to an extremely weak, non-detectable fluorescence. It is known that the obtained fluorescence intensity, with a few exceptions, is directly correlated with the growth rate of the target bacteria. The accessibility of the targets is controlled mainly by cell wall properties, which again require to get permeabilized by either the fixative or, in case of gram positive cells, lysozyme [25]. As P. intermedia and streptococci were readily stained at the base of the biofilms, a

Table 1 Growth media

\begin{tabular}{|c|c|c|c|}
\hline Medium & Abbreviation & Reference & Use \\
\hline mFUM, 4 mM Glucose & mFUM4 & & Growth medium for biofilms \\
\hline mFUM 4 mM Glucose, iHS (50\%) & $i \mathrm{HS}$ & & Growth medium for biofilms \\
\hline $\begin{array}{l}\text { mFUM, 0.3\% Glucose (30\%), saliva (60\%), } \\
\text { iHS (10\%) }\end{array}$ & SAL & & Growth medium for biofilms \\
\hline mFUM, $0.3 \%$ Glucose & & [12] & $\begin{array}{l}\text { Liquid precultures of S. oralis, S. anginosus, V. dispar }{ }^{1} \text {, F. nucleatum, A. oris, } P \text {. } \\
\text { intermedia, C. rectus }^{2}\end{array}$ \\
\hline Pg medium $^{3}$ & & [29] & Liquid precultures of $P$. gingivalis \\
\hline Spirochaetes medium & & {$[30]$} & Precultures of $T$. denticola \\
\hline Modified OMIZ-W68 ${ }^{4}$ & & {$[31]$} & Precultures of $T$. forsythia \\
\hline
\end{tabular}

1 addition of $1 \%$ lactic acid ( $/ \mathrm{v})$.

2 addition of $0.1 \%$ sodium fumarate and $0.1 \%$ sodium formiate.

${ }^{3}$ Brain heart infusion broth, supplemented with haemin $(7.67 \mu \mathrm{M})$ and menadione $(2.91 \mu \mathrm{M})$.

${ }^{4}$ addition of lactose $\left(2 \mathrm{~g} \mathrm{I}^{-1}\right)$, caseinoglycomacropeptide $\left(100 \mathrm{mg} \mathrm{l}^{-1}\right), \mathrm{N}$-acetylmuramic acid $\left(50 \mathrm{mg} \mathrm{l}^{-1}\right)$, and $\mathrm{N}$ - acetylglucosamine $\left(500 \mathrm{mg} \mathrm{l}{ }^{-1}\right)$. 
Table 2 Sequences, labels and formamide concentrations for FISH Probes

\begin{tabular}{|c|c|c|c|c|c|c|c|}
\hline Organism & Name & Type & Labels & $\mathrm{FA}^{1}$ & $W^{2}$ & Sequence $\left(5^{\prime} \rightarrow 3^{\prime}\right)$ & References \\
\hline A. oris & L-Act476-2 & $\mathrm{LNA}^{3}$ & Cy3, FAM, 6-Rox & $40 \%$ & $46 \mathrm{mM}$ & ATCCAGCTACCGTCAACC & [11] \\
\hline C. rectus & CAMP665 & DNA & Cy3, Cy5 & $30 \%$ & $112 \mathrm{mM}$ & CATCTGCCTCTCCCTYAC & [11] \\
\hline \multirow[t]{2}{*}{ F. nucleatum } & FUS664 & & Cy3, Cy5, FAM, FITC & $40 \%$ & $46 \mathrm{mM}$ & CTTGTAGTTCCGCYTACCTC & [32] \\
\hline & Fnuc133c & DNA & Cy3, Cy5 & $40 \%$ & $46 \mathrm{mM}$ & GTTGTCCCTANCTGTGAGGC & [11] \\
\hline P. intermedia & L-Pint649-2 & LNA & Cy3, FAM,6-Rox & $40 \%$ & $46 \mathrm{mM}$ & CGTTGCGTGCACTCAAGTC & [11] \\
\hline P. gingivalis & L-Pgin1006-2 & $\mathrm{LNA}^{3}$ & Cy3, Cy5, FAM & $30 \%$ & $112 \mathrm{mM}$ & GTITCACCATCMGTCATC & [11] \\
\hline Streptococci & STR405 & DNA & Cy3, Cy5 & $20 \%$ & $215 \mathrm{mM}$ & TAGCCGTCCCTTTCTGGT & [34] \\
\hline T. denticola & TrepG1_679 & DNA & Cy3, Cy5, FAM & $40 \%$ & $46 \mathrm{mM}$ & GATTCCACCCCTACACTT & [13] \\
\hline T. forsythia & Tfor997 & DNA & Cy3, Cy5, FAM & $40 \%$ & $46 \mathrm{mM}$ & TCACTCTCCGTCGTCTAC & [35] \\
\hline V. dispar & VEI217 & DNA & Cy3, Cy5, FAM, FITC, 6-Rox & $40 \%$ & $46 \mathrm{mM}$ & AATCCCCTCCTTCAGTGA & [32] \\
\hline
\end{tabular}

${ }^{1}$ Formamide concentration in the hybridisation buffer

${ }^{2}$ Concentration of $\mathrm{NaCl}$ used in the washing buffer.

${ }^{3}$ Probes containing locked nucleic acid substitutes (LNA). The ribose ring of LNA is constrained by a methylene linkage between the 2 ' oxygen and the 4 ' carbon.

hindered diffusion of the probes or fixatives through the biofilms does not seem to be the problem. The accessibility of the cells can be sorted out as well, as the signal is very clear in the top layer of the biofilm. Careful examination of the images, by enhancing the contrast settings for the general DNA staining in our samples, revealed structures at the base of the biofilms that very much resembles the well-stained colonies of $F$. nucleatum observed in less thick biofilms. Combined with the high abundance detected by IF, it seems that $F$. nucleatum was in fact present in at the base of the biofilms, however, either in a non-viable- or at least non-active state. For future experiments, it might be worth investigating new methods to increase fluorescent signals, in order to obtain a bright staining throughout the whole biofilm. Catalysed reporter deposition (CARD)-FISH [26], the use of helper oligonucleotides [27], or designing probes targeting the 23S rRNA [28] might be solutions. Due to the large size of the horseradish peroxidase used with CARD-FISH, it seems unlikely that this method would be appropriate, and the use of helper oligonucleotides or probes targeting the 23S rRNA seem more promising to reach stronger signals.

One of the major differences to the in vivo situation is that the model biofilms grew without the presence of an epithelial cell layer. Some of the observed differences will be caused by the lack of interactions that occur in vivo. A future project will address this circumstance and aims to incorporate an epithelial cell layer into the model system. The main difficulty in maintaining such a co-culture system is that different growth conditions that are needed to cultivate either epithelial cells or biofilms. While the strict anaerobes in the consortium of the biofilms are very sensitive to oxygen, the epithelial cells do require oxygen for growth. Further, biofilms and epithelial cells do have very different nutritional requirements. In our co-culture experiments performed so far, cells and biofilms were cultured separately and incubated as co-culture after the development of both biofilms and epithelial cells [11]. Current experiments showed, that the biofilm consortium is still able to grow on agar plates after $48 \mathrm{~h}$ of co-culture, however, the viability of the bacteria was greatly reduced (data not shown).

\section{Conclusions}

We were able to locate all 10 species by CLSM and reconstruct the three-dimensional structure of our model biofilms (Figure 9). The structural analysis revealed a close proximity of $T$. denticola and $P$. gingivalis in the top layer of the biofilms, which might indicate a high pathogenic potential of these in vitro formed subgingival model biofilms. $V$. dispar appeared in the top layer as well, forming tight microcolonies.

The use of $50 \%$ heat-inactivated human serum in the growth medium improved the stability of the biofilms, resulting in significantly thicker biofilms. Under these conditions the fastidious $T$. denticola was able to establish in significantly higher densities compared to the media with $10 \%$ or no human serum. Surprisingly, neither P. gingivalis nor $T$. forsythia were affected by the concentration of human serum, and neither by the addition of saliva.

Table 3 Antibodies used for IF

\begin{tabular}{llll}
\hline Target & Cell Line/MAb & Isotype & Reference \\
\hline C. rectus & 212WR2 & mouse lgG3 & {$[36]$} \\
T. forsythia & $103 \mathrm{BF} 1.1$ & mouse $\operatorname{lgG} 2 \mathrm{~b}$ & {$[37]$} \\
P. gingivalis & $61 \mathrm{BG} 1.3$ & mouse $\operatorname{lgG} 1$ & {$[38]$} \\
P. intermedia & $37 \mathrm{Bl6} .1$ & rat lgG2b & {$[39]$} \\
F. nucleatum & 305FN1.2 & mouse lgM & {$[40]$} \\
A. oris & 396AN1 & mouse lgM & {$[41]$} \\
\hline
\end{tabular}




\section{Methods}

\section{Biofilm generation and fixation}

The biofilms used in this study are produced using a similar protocol as described before [11]. However, there are some key changes in the growth media and the strain composition that are described below. In the present study, Streptococcus oralis SK248 (OMZ 607), Streptococcus anginosus ATCC 9895 (OMZ 871), Actinomyces oris (OMZ 745; formerly Actinomyces naeslundii), Fusobacterium nucleatum subsp. nucleatum OMZ 598, Veillonella dispar ATCC $17748^{\mathrm{T}}$ (OMZ 493), Campylobacter rectus OMZ 698, Prevotella intermedia ATCC $25611^{\mathrm{T}}(\mathrm{OMZ}$ 278), Porphyromonas gingivalis ATCC $33277^{\mathrm{T}}$ (OMZ 925), Tannerella forsythia OMZ 1047, and Treponema denticola ATCC $35405^{\mathrm{T}}$ (OMZ 661) were used.

All strains, except for $T$. forsythia and $C$. rectus, were maintained on Columbia blood agar (CBA). T. forsythia and $T$. denticola were maintained in liquid culture using the media outlined in Table 1. Prior to the onset of biofilm experiments, all strains were transferred into adequate liquid media (Table 1) for two cycles of precultures. The slow growing $T$. forsythia, $C$. rectus and $T$. denticola were precultured for $64 \mathrm{~h}$ (first cycle), then diluted 1:2 in fresh media and incubated for another $24 \mathrm{~h}$ (second cycle). All other strains were incubated over night (first cycle), diluted 1:10 in fresh media and incubated again for $8 \mathrm{~h}$ (second cycle). Prior to biofilm inoculation, all strains were adjusted to a defined optical density $\left(\mathrm{OD}_{550}=1.0\right.$ except for $C$. rectus, $T$. denticola with $\left.\mathrm{OD}_{550}=0.5\right)$ and mixed in equal volumes. Sintered circular HA discs with a diameter of $10.6 \mathrm{~mm}$ (Clarkson Chromatography Products, South Williams-port, USA) were coated with 1:2 diluted saliva for pellicle formation. Discs were placed in 24-well polystyrene cell culture plates and covered with $1.5 \mathrm{ml}$ of growth medium. In this study three different growth media, all based on mFUM [12], were used (Table 1). For inoculation $200 \mu \mathrm{l}$ of the mixed precultures were added. Incubation was anaerobic and lasted $64.5 \mathrm{~h}$. The medium was renewed after $16.5 \mathrm{~h}$ and subsequently every $24 \mathrm{~h}$. After the first renewal of growth media, each well was supplemented with a boost of $40 \mu \mathrm{l}$ of $T$. denticola liquid culture $\left(\mathrm{OD}_{550}=0.5\right)$. Biofilms were dip-washed three times daily at intervals of 3$4 \mathrm{~h}$. For dip-washings the discs were placed in $0.9 \% \mathrm{NaCl}$ and washed by gentle agitation for 45 seconds. After this step, the discs were dipped twice two times each in two wells of fresh saline. Then the discs were returned to medium for further incubation.

For confocal microscopy, biofilms were fixed directly on the discs for at least $1 \mathrm{~h}$ at $4^{\circ} \mathrm{C}$ in $4 \%$ paraformaldehyde (Merck, Darmstadt, Germany) after the last dipwash. For quantification by microscopic counting, biofilms were removed from the discs by vortexing ( $2 \mathrm{~min}$ in a $50 \mathrm{ml}$ tube with $1 \mathrm{ml}$ of in $0.9 \% \mathrm{NaCl}$ ) and sonicated for $5 \mathrm{sec}$ at $25 \mathrm{~W}$ (Branson Sonic Power Company, Sonifier B-12) to reduce cell aggregation and the processed as described below.

\section{FISH staining procedure}

The FISH procedure was done using the same conditions for the hybridisation as described by Thurnheer et al. [32]. Probe sequences, formamide concentrations used for the hybridisations, as well as the $\mathrm{NaCl}$ concentrations of the washing buffers are given in Table 2. To hybridise gram-positive bacteria, biofilms were pretreated in lysozyme solution with a concentration of 1 $\mathrm{mg} / \mathrm{ml}$ lysozyme (5 $\mathrm{min}$, room temperature). The lysozyme solution consisted of $1 \mathrm{mg}$ lysozyme from chicken egg white containing 70'000 units/mg (Fluka), dissolved in $890 \mu \mathrm{l} \mathrm{H} \mathrm{H}_{2} \mathrm{O}, 100 \mu \mathrm{l} 1 \mathrm{M}$ Tris- $\mathrm{HCl}$ solution (ICN Biomedicals, Inc.), $\mathrm{pH}=7.5$, and $10 \mu \mathrm{l} 0.5 \mathrm{M}$ EDTA solution (Fluka), $\mathrm{pH}=8.0$. If the combination of probes required different formamide concentrations, the hybridisations were performed consecutively, starting with the highest concentration. Pre-hybridisation $\left(15 \mathrm{~min}, 46^{\circ} \mathrm{C}\right)$ was performed in $500 \mu \mathrm{l}$ hybridisation buffer without probes added. $500 \mu \mathrm{l}$ of hybridisation buffer was used for each biofilm, supplemented with of up to 3 probes at a concentration of $10 \mathrm{ng} / \mu \mathrm{l}$ for each. The incubation time for the hybridisation was at least $3 \mathrm{~h}$ at $46^{\circ} \mathrm{C}$ in the dark. After the incubation, biofilms were transferred into washing buffer pre-heated to $48^{\circ} \mathrm{C}$ and incubated for 20 $\min$ at $48^{\circ} \mathrm{C}$. For counterstaining, biofilms were stained using a mixture of $3 \mu \mathrm{M}$ YoPro- 1 iodide (Invitrogen) and $15 \mu \mathrm{M}$ Sytox green (Invitrogen) $(20 \mathrm{~min}$, room temperature, in the dark) following the FISH procedure. To stain EPS, calcofluor (Sigma Chemical, Buchs, Switzerland); $10 \mu \mathrm{g} / \mathrm{ml}$ solution in $10 \mathrm{mM}$ sodium phosphate, $\mathrm{pH}$ 7.5) was applied parallel to the counterstaining. After hybridisation the samples were embedded upside down on chamber slides in $100 \mu \mathrm{l}$ of Mowiol [33].

\section{Quantification of FISH- and IF-stained bacteria}

Harvested biofilms were quantified microscopically using FISH and IF. Samples were serially diluted, mounted and fixed on 24-well slides as described by Züger et al. [35]. $S$. oralis, $S$. anginosus, $T$. denticola and $V$. dispar were stained by FISH using the probes listed in Table 2, while $C$. rectus, $T$. forsythia, $P$. gingivalis, $P$. intermedia, $F$. nucleatum and $A$. oris were stained by IF using the monoclonal antibodies listed in Table 3. The protocols for FISH and IF, and the counting were as described by Züger et al. [35].

\section{Structural analysis}

Biofilms were stained directly on the hydroxyapatite (HA) discs by multiplex FISH and analysed by confocal laser scanning microscopy (CLSM) [32]. A Leica SP-5 microscope with resonant scanner system $(8000 \mathrm{~Hz})$, provided 
by the Centre of Microscopy and Image Analysis of the University of Zürich (ZMB), was used for imaging the biofilms. All images were captured using a 63x objective (glycerol immersion, NA 1.3). The system was equipped with a diode laser (405 nm excitation), an argon laser $(458 \mathrm{~nm} /$ $476 \mathrm{~nm} / 488 \mathrm{~nm} / 496 \mathrm{~nm} / 514 \mathrm{~nm}$ excitation) and a helium neon laser $(561 \mathrm{~nm} / 594 \mathrm{~nm} / 633 \mathrm{~nm}$ excitation). The laser settings varied depending on the used combination of probe labels (Cy3, Cy5, 6-Rox) and optimal settings were obtained using the spectra settings of the Leica software and/or the Invitrogen Fluorescence SpectraViewer (http:// www.invitrogen.com/site/us/en/home/support/ResearchTools/Fluorescence-SpectraViewer.html) to adjust the settings manually. The thickness of the biofilms was determined using the $\mathrm{xz}$ view, and the measurement was performed using the measurement tool incorporated in the Leica software. For the creation of the stacked sliceand 3D - images, Imaris (Bitplane) was used.

\section{Statistical evaluation}

All data presented in this study derive from three independent experiments. In each experiment, biofilms were cultured in triplicates for each examined time point and for each growth medium. Total counts presented in Figure 1 were determined by counting of colony forming units on CBA agar, while the total counts shown in Figure 3 were calculated based on the species-specific quantification by FISH and IF. One additional disc for each growth medium and time point was used to measure the thickness of the biofilms by CLSM. Using the logarithmized values of the abundances $(\mathrm{N}=9$ values for each species), the Kruskal-Wallis test with $p \leq 0.05$ was performed to determine the significance levels given in Figure 4 . The thickness of the biofilms was measured on 9 independent biofilms, with $\mathrm{N}=44$ measurements on iHS biofilms, $\mathrm{N}=61$ on mFUM4 biofilms, and $\mathrm{N}=57$ on SAL biofilms. Significance was tested by ANOVA (Bonferroni test with $\mathrm{p} \leq 0.001$ ).

\section{Abbreviations \\ EPS: Exopolysaccharide; mFUM: Modified fluid universal medium; FISH: Fluorescence in situ hybridisation; IF: Immunofluorescence; CLSM: Confocal laser scanning microscopy.}

\section{Authors' contributions}

TWA designed the study, executed the experiments and drafted the manuscript. TT and RG supervised the study and edited the manuscript draft. All authors read and approved the final manuscript.

\section{Acknowledgements}

We thank Ruth Graf and Andy Meier for their support with the maintenance of the bacteria as well as the cultivation of the biofilms, and Helga LüthiSchaller for her assistance with FISH and IF. We thank the Centre of Microscopy and Image Analysis (ZMB) of the University of Zürich for their support with confocal microscopy. TWA was supported by grant 242-09 from the research fund of the Swiss Dental Association (SSO).

Received: 13 April 2012 Accepted: 4 October 2012

Published: 5 October 2012

\section{References}

1. Flemming HC: The perfect slime. Colloid Surface B 2011, 86:251-259.

2. Jenkinson HF: Beyond the oral microbiome. Environ Microbiol 2011 , 13:3077-3087

3. Marsh PD, Percival RS: The oral microflora - friend or foe? Can we decide? Int Dent J 2006, 56:233-239.

4. Van Dyke TE, Sheilesh D: Risk factors for periodontitis. J Int Acad Periodontol 2005, 7:3-7.

5. Li XJ, Kolltveit KM, Tronstad L, Olsen I: Systemic diseases caused by oral infection. Clin Microbiol Rev 2000, 13:547-558.

6. Socransky SS, Haffajee AD: Dental biofilms: difficult therapeutic targets. Periodontol 2002, 28:12-55.

7. Paster BJ, Olsen I, Aas JA, Dewhirst FE: The breadth of bacterial diversity in the human periodontal pocket and other oral sites. Periodontol 2006, 42:80-87.

8. Zijnge V, Ammann T, Thurnheer T, Gmür R: In Subgingival biofilm structure, Frontiers of Oral Biology. Edited by Mombelli A, Kinane DF. Basel: Karger; 2012:1-16.

9. Shaddox LM, Alfant B, Tobler J, Walker C: Perpetuation of subgingival biofilms in an in vitro model. Mol Oral Microbiol 2010, 25:81-87.

10. Hope CK, Wilson M: Biofilm structure and cell vitality in a laboratory model of subgingival plaque. J Microbiol Methods 2006, 66:390-398.

11. Guggenheim B, Gmür R, Galicia JC, Stathopoulou PG, Benakanakere MR, Meier A, Thurnheer T, Kinane DF: In vitro modeling of host-parasite interactions: the 'subgingival' biofilm challenge of primary human epithelial cells. BMC Microbiol 2009, 9:280.

12. Guggenheim B, Giertsen E, Schupbach P, Shapiro S: Validation of an in vitro biofilm model of supragingival plaque. J Dent Res 2001, 80:363-370

13. Zijnge $V$, van Leeuwen MB, Degener JE, Abbas F, Thurnheer T, Gmür R, Harmsen HJ: Oral biofilm architecture on natural teeth. PLOS One 2010, 5:e9321.

14. Kolenbrander PE, London J: Adhere today, here tomorrow: oral bacterial adherence. J Bacterio/ 1993, 175:3247-3252.

15. Ruiz V, Rodriguez-Cerrato V, Huelves L, Del Prado G, Naves P, Ponte C, Soriano F: Adherence of streptococcus pneumoniae to polystyrene plates and epithelial cells and the antiadhesive potential of albumin and xylitol. Pediatr Res 2011, 69:23-27.

16. Naves P, del Prado G, Huelves $L$, Rodriguez-Cerrato V, Ruiz V, Ponte MC, Soriano F: Effects of human serum albumin, ibuprofen and N-acetyl-Lcysteine against biofilm formation by pathogenic Escherichia coli strains. J Hosp Infect 2010, 76:165-170.

17. Hojo K, Nagaoka S, Ohshima T, Maeda N: Bacterial interactions in dental biofilm development. J Dent Res 2009, 88:982-990.

18. Wyss C: Growth of Porphyromonas gingivalis, Treponema denticola, T. pectinovorum, T. socranskii, and T. vincentii in a chemically defined medium. J Clin Microbiol 1992, 30:2225-2229.

19. Thurnheer T, Gmür R, Shapiro S, Guggenheim B: Mass transport of macromolecules within an in vitro model of supragingival plaque. Appl Environ Microbiol 2003, 69:1702-1709.

20. Kesavalu L, Holt SC, Ebersole JL: Virulence of a polymicrobic complex Treponema denticola and Porphyromonas gingivalis, in a murine model. Oral Microbiol Immun 1998, 13:373-377.

21. Orth RK, O'Brien-Simpson NM, Dashper SG, Reynolds EC: Synergistic virulence of Porphyromonas gingivalis and Treponema denticola in a murine periodontitis model. Mol Oral Microbiol 2011, 26:229-240.

22. Grenier D: Nutritional Interactions between Two Suspected Periodontopathogens, Treponema denticola and Porphyromonas gingivalis. Infect Immun 1992, 60:5298-5301.

23. Ito R, Ishihara K, Shoji M, Nakayama K, Okuda K: Hemagglutinin/Adhesin domains of Porphyromonas gingivalis play key roles in coaggregation with Treponema denticola. FEMS Immunol Med Microbiol 2010, 60:251-260.

24. Sharma A, Inagaki S, Sigurdson W, Kuramitsu HK: Synergy between Tannerella forsythia and Fusobacterium nucleatum in biofilm formation. Oral Microbiol Immunol 2005, 20:39-42.

25. Amann Rl, Ludwig W, Schleifer KH: Phylogenetic identification and in situ detection of individual microbial cells without cultivation. Microbio/ Rev 1995, 59:143-169.

26. Pernthaler A, Pernthaler J, Amann R: Fluorescence in situ hybridization and catalyzed reporter deposition for the identification of marine bacteria. Appl Environ Microbiol 2002, 68:3094-3101. 
27. Fuchs BM, Glockner FO, Wulf J, Amann R: Unlabeled helper oligonucleotides increase the in situ accessibility to 16S rRNA of fluorescently labeled oligonucleotide probes. Appl Environ Microbiol 2000, 66:3603-3607.

28. Fuchs BM, Syutsubo K, Ludwig W, Amann R: In situ accessibility of Escherichia coli $23 \mathrm{~S}$ rRNA to fluorescently labeled oligonucleotide probes. Appl Environ Microbiol 2001, 67:961-968.

29. Milner $\mathrm{P}$, Batten JE, Curtis MA: Development of a simple chemically defined medium for Porphyromonas gingivalis: requirement for alphaketoglutarate. FEMS Microbiol Lett 1996, 140:125-130.

30. Blakemore RP, Canale-Parola E: Arginine catabolism by Treponema denticola. J Bacteriol 1976, 128:616-622.

31. Wyss C: Fatty acids synthesized by oral treponemes in chemically defined media. FEMS Microbiol Lett 2007, 269:70-76.

32. Thurnheer T, Gmür R, Guggenheim B: Multiplex FISH analysis of a sixspecies bacterial biofilm. J Microbiol Methods 2004, 56:37-47.

33. Guggenheim M, Shapiro S, Gmür R, Guggenheim B: Spatial arrangements and associative behavior of species in an in vitro oral biofilm model. Appl Environ Microbiol 2001, 67:1343-1350.

34. Thurnheer T, Gmur R, Giertsen E, Guggenheim B: Automated fluorescent in situ hybridization for the specific detection and quantification of oral streptococci in dental plaque. J Microbiol Methods 2001, 44:39-47.

35. Züger J, Lüthi-Schaller H, Gmür R: Uncultivated Tannerella BU045 and BU063 are slim segmented filamentous rods of high prevalence but low abundance in inflammatory disease-associated dental plaques. Microbiology 2007, 153:3809-3816.

36. Gmür R: Value of new serological probes for the study of putative periodontal pathogens. Zurich: Dental Center of the University of Zurich; 1995:86.

37. Werner-Felmayer G, Guggenheim B, Gmür R: Production and characterization of monoclonal antibodies against Bacteroides forsythus and Wolinella recta. J Dent Res 1988, 67:548-553.

38. Gmür R, Werner-Felmayer $G$, Guggenheim B: Production and characterization of monoclonal antibodies specific for Bacteroides gingivalis. Oral Microbiol Immunol 1988, 3:181-186.

39. Gmür R, Guggenheim B: Antigenic heterogeneity of Bacteroides intermedius as recognized by monoclonal antibodies. Infect Immun 1983, 42:459-470.

40. Thurnheer T, Guggenheim B, Gruica B, Gmür R: Infinite serovar and ribotype heterogeneity among oral Fusobacterium nucleatum strains? Anaerobe 1999, 5:79-92.

41. Thurnheer T, Guggenheim B, Gmür R: Characterization of monoclonal antibodies for rapid identification of Actinomyces naeslundii in clinical samples. FEMS Microbiol Lett 1997, 150:255-262.

doi:10.1186/1471-2180-12-227

Cite this article as: Ammann et al: Advancement of the 10-species subgingival Zurich Biofilm model by examining different nutritional conditions and defining the structure of the in vitro biofilms. BMC Microbiology 2012 12:227.

\section{Submit your next manuscript to BioMed Central and take full advantage of:}

- Convenient online submission

- Thorough peer review

- No space constraints or color figure charges

- Immediate publication on acceptance

- Inclusion in PubMed, CAS, Scopus and Google Scholar

- Research which is freely available for redistribution 